\title{
Effect of hypoxia-inducible factor-1/vascular endothelial growth factor signaling pathway on spinal cord injury in rats
}

\author{
HAILONG CHEN*, JUNJIE LI*, SHUHAN LIANG, BIN LIN, QI PENG, \\ PENG ZHAO, JIAWEI CUI and YAOJIAN RAO
}

\author{
Department of Spine Surgery, Luoyang Orthopedic Hospital of Henan, Luoyang, Henan 471002, P.R. China
}

Received September 2, 2015; Accepted October 4, 2016

DOI: $10.3892 / \mathrm{etm} .2017 .4049$

\begin{abstract}
The aim of the present study was to evaluate the expression of vascular endothelial growth factor (VEGF) and hypoxia inducible factor-1 (HIF-1), and to investigate the role of the HIF-1/VEGF signaling pathway following spinal cord injury (SCI). A total of 90 12-week-old Sprague Dawley rats were randomly divided into the following three groups: Sham group (operation without SCI); control group (SCI without ML228 treatment); and treatment group (SCI receiving ML228 treatment). ML228 was administered as it is an activator of HIF-1 $\alpha$. The control and treatment groups were subjected to spinal cord hemisection and motor activity was evaluated using the Basso, Beattie and Bresnahan (BBB) scoring system. Expression of HIF-1 $\alpha$ and VEGF in each injured spinal cord section was assessed using immunohistochemistry. Prior to $\mathrm{SCI}$, there were no significant differences in the BBB score among the three groups $(\mathrm{P}>0.05)$. However, one day after the operation, the BBB score of the sham group was significantly higher than that of the other two groups $(\mathrm{P}<0.05)$ and the BBB scores of the control and treatment groups did not differ significantly $(\mathrm{P}>0.05)$. BBB scores 3 and 7 days following surgery were significantly higher in the sham group than the other two groups $(\mathrm{P}<0.05)$ and the BBB scores of the treatment group were significantly higher than those of the control group $(\mathrm{P}<0.05)$. The expression of HIF-1 $\alpha$ and VEGF proteins in all groups were measured 1, 3 and 7 days after the operation, and it was observed that their expression was higher in the treatment group than in the control group $(\mathrm{P}<0.05)$. Therefore, the results of the current study suggest that ML228 may effectively activate the HIF-1 $\alpha$ /VEGF signaling pathway to promote the expression of HIF-1 $\alpha$ and VEGF proteins within
\end{abstract}

Correspondence to: Dr Yaojian Rao, Department of Spine Surgery, Luoyang Orthopedic Hospital of Henan, 82 Qimingnan Road, Luoyang, Henan 471002, P.R. China

E-mail:raorao5069@sina.com

*Contributed equally

Key words: hypoxia inducible factor $1 /$ vascular endothelial growth factor signaling pathway, ML228, spinal cord injury the injured segment of the spinal cord, which promotes neural functional recovery following SCI in rats. Therefore, treatment with ML228 may be developed as a novel therapeutic strategy to treat SCI.

\section{Introduction}

Spinal cord injury (SCI) is the primary cause of paraplegia. Nerve regeneration and restoration is considered extremely difficult and is a major focus of current neuroscience research. The secondary mechanisms that occur following SCI include enclosed blood circulation obstacles, changes in the levels of biologically active substances and the development of energy metabolic disorders (1). However, one popular hypothesis is that the primary problem resulting from secondary spinal injury is chemical hypoxia (2), and further damage following SCI is caused by decreased local blood flow and hypoxia. It has been demonstrated that hypoxia inducible factor 1 (HIF-1) is specific and sensitive to the hypoxia signaling response (3).

As well as the characteristics of its existence and the regulation of target genes, HIF-1 is considered to be the low oxygen related regulatory gene in the core of the most important hypoxia-related transcription factors (4). When tissues or cells are in a hypoxic environment, HIF-1 is one of the most important transcription factors regulating oxygen metabolism (5). HIF-1 expression is increased following hypoxia and may specifically bind to the hypoxia response element of the vascular endothelial growth factor (VEGF) promoter (6), strengthening the stability of the biological functions of VEGF and promoting VEGF expression, transcription and translation. The primary function of VEGF in the nervous system is associated with angiogenesis, and by promoting angiogenesis and improving local microcirculation, VEGF may indirectly protect the nerve from ischemic and anoxic injury (7).

At present, research into the role that HIF-1/VEGF signaling pathways serve in the response to SCI is very limited. ML228 was selected for further characterization as it exhibited the most improved potency within the chemotype and was considered to be an interesting tool compound. ML228 represents a novel chemotype available to the research community for the study of HIF activation and its therapeutic potential. Not only is the compound substantially different in structure from other known HIF activators, ML228 also lacks the acidic functional group almost universally present 
in prolyl hydroxylase inhibitors, which may be important for certain disease applications. ML228 was demonstrated to potently activate HIF in vitro as well as its downstream target, VEGF (8). Therefore, the present study evaluated the effect of ML228, which has been shown to activate HIF as well as its downstream target VEGF (9), on the HIF-1/VEGF signaling pathway following the induction of SCI in a rat model. In addition, the effect of the activation of this signalling pathway on the response to SCI was investigated. Therefore, the effect of ML228 on recovery from SCI was evaluated in the current study.

\section{Materials and methods}

Animals and reagents. A total of 90 Sprague Dawley rats (female, 12 weeks old; weight, 220-255 g) were purchased from the SCXK Experimental Animal Center of Henan Province (Zhengzhou, China). Antibodies for HIF-1 $\alpha$ (BA0912-2) and VEGF (PB0084), and goat anti-mouse secondary antibody (BA1051), were purchased from Wuhan Boster Biotechnology, Ltd. (Wuhan, China). Hematoxylin and eosin (H\&E) dye was purchased from Nanjing Jiancheng Technology, Co., Ltd. (Nanjing, China). The specific activator of HIF-1/VEGF signaling pathways ML228 (10) was purchased from MedChemExpress China (Shanghai, China).

Animal model. Sprague Dawley rats were randomly divided into the following three groups $(n=30$ each): Sham group; the control group (SCI without ML228 treatment); and treatment group (SCI rats that received $1 \mu \mathrm{g} / \mathrm{kg}$ ML228 treatment). All rats were anaesthetized by intraperitoneal injection of cocktail (2 ml/kg) of xylazine $(1.3 \mathrm{mg} / \mathrm{ml}$; X1126; Sigma-Aldrich; Merck Millipore, Darmstadt, Germany), ketamine (25 mg/ml; 16519-5; Cayman Chemical Company, Ann Arbor, MI, USA) and acepromazine $(0.25 \mathrm{mg} / \mathrm{ml}$; A7111; Sigma-Aldrich; Merck Millipore). Following back shaving and sterilization, an incision was made on the back posterior to the lower thoracic region. When the back muscles had been infiltrated, laminectomy at the T10 level exposed the dorsal surface of the spinal cord and the lower thoracic cord was subsequently transected using fine scissors. Finally, the surgical wound was closed in two layers. Sham rats underwent a similar operation to rats in the SCI groups, with the exception that the lower thoracic cord was exposed but not transected. Daily assistance in bladder emptying was given to rats that had undergone SCI until spontaneous miction recovered. All rats had ad libitum access to food and water, and were fed with commercial rat chow comprising $0.95 \%$ calcium and $0.67 \%$ phosphate. Rats were housed in a controlled environment at $22^{\circ} \mathrm{C}$ and $50 \%$ humidity with a $12 \mathrm{~h} \mathrm{light/dark} \mathrm{cycle.} \mathrm{To} \mathrm{prevent} \mathrm{infection,} \mathrm{all} \mathrm{rats}$ received an intramuscular injection of 80,000 units penicillin every day for 7 days. One rat succumbed to mortality on the first day; therefore, a new rat was added to maintain the total number of 90. All experiments were completed according to the protocols of the University Animal Welfare and Ethical Review Committees of Zheng Gu hospital.

Behavioral testing. Basso, Beattie and Bresnahan (BBB) scores were assigned on the day prior to operation, and 1,3 and 7 days following operation (10). All rats were randomly assigned to groups, ensuring that initial locomotor scores were equalized among the groups. Severe compression injury produces spontaneous recovery to a BBB score of 4 (11). Behavioral testing was performed daily by two individuals blinded to the treatment groups, and functional recovery was assessed using the BBB locomotor rating scale. To determine whether these functional recoveries were due to axonal regeneration or activated local reflex of the segment below the injured site, transections on all rats were performed one segment distal (T10) from the previously injured spinal level. An anesthesia cocktail $(2 \mathrm{ml} / \mathrm{kg}$ ) of ketamine, xylazine and acepromazine was administered, and the transection was performed as described previously (12). Samples were frozen at $-80^{\circ} \mathrm{C}$ prior to further experiments. Rats were sacrificed using an overdose of pentobarbital sodium (100 mg/kg via intraperitoneal injection; P3761; Sigma-Aldrich; Merck Millipore) followed by transcardial perfusion.

$H \& E$ staining. H\&E staining was performed as follows: Frozen sections of spinal cord (thickness, $2 \mu \mathrm{g}$ ) were dried in air following polysine coating, washed in distilled water for 1-2 min, immersed in hematoxylin solution for $1 \mathrm{~min}$ and washed again. Following treatment with $1 \%$ hydrochloric acid for $3 \mathrm{sec}$, sections were washed and treated with saturated lithium carbonate solution for $1 \mathrm{~min}$, followed by washing. Then, sections were dehydrated in $80 \%$ alcohol for 1-2 min, stained in eosin for 1-2 min and subsequently washed. Following dehydration in graded alcohol and transparentization in xylene, sections were mounted with neutral gum. Sections were observed using light microscopy (DMLP-MP30; Leica Microsystems GmbH, Wetzlar, Germany).

Western blot analysis. Transverse sections of spinal cords were lysed using radioimmunoprecipitation assay buffer (AR0105-10; Wuhan Boster Biotechnology, Ltd.) following treatment. Lysates $(20 \mu \mathrm{l} /$ lane $)$ were separated by $10 \%$ SDS-PAGE and transferred to PVDF membranes. Membranes were probed with primary anti-HIF-1 $\alpha$ and anti-VEGF antibodies (both 1:500), and goat anti-mouse secondary antibody $(1: 1,000)$ and treated with chemiluminescence detection kit (A3417_5000-1; AppliChem GmbH, Darmstadt, Germany) according to the manufacturer's protocol. Band intensities were quantified using Image $\mathbf{J}$ (http://imagej.net/). $\beta$-actin (1:500; BM0627; Wuhan Boster Biotechnology, Ltd.) was used as a reference.

Immunohistochemistry. On 1, 3, 7 day following SCI or sham operation, 10 rats per group were sacrificed using an overdose of pentobarbital sodium (100 mg/kg via intraperitoneal injection), followed by transcardial perfusion of $0.9 \%$ saline for $5 \mathrm{~min}$ and then $4 \%$ paraformaldehyde (Sigma-Aldrich; Merck Millipore)

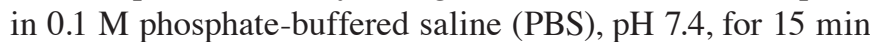
at room temperature. Two 3-mm spinal cord segments, $1.5 \mathrm{~mm}$ rostral and $1.5 \mathrm{~mm}$ caudal to the lesion epicenter, were removed from each rat and post-fixed in the same fixative for $2 \mathrm{~h}$ at $4^{\circ} \mathrm{C}$. Subsequently, segments were cryopreserved in $30 \%$ sucrose solution in PBS for 2-3 days at $4^{\circ} \mathrm{C}$. Spinal cords were frozen in Tissue-Tek ${ }^{\circledR}$ optimum cutting temperature compound (Sakura Finetek USA, Inc., Torrance, CA, USA) and $15 \mu \mathrm{m}$ transverse sections were cut and mounted on gelatin-coated glass slides. 
Table I. Basso, Beattie, and Bresnahan scores at different time points $(n=90)$.

\begin{tabular}{lcccc}
\hline Group & Prior to operation & 1 day after operation & 3 days after operation & 7 days after operation \\
\hline Sham group $(\mathrm{n}=30)$ & $21.00 \pm 0.00$ & $18.34 \pm 0.42$ & $18.96 \pm 0.33$ & $19.34 \pm 0.41$ \\
Treatment group $(\mathrm{n}=30)$ & $21.00 \pm 0.00$ & $1.51 \pm 0.55^{\mathrm{a}, \mathrm{b}}$ & $4.37 \pm 0.51^{\mathrm{a}, \mathrm{b}}$ & $8.49 \pm 0.43^{\mathrm{a}, \mathrm{b}}$ \\
Control group $(\mathrm{n}=30)$ & $21.00 \pm 0.00$ & $1.84 \pm 0.47^{\mathrm{a}}$ & $2.09 \pm 0.44^{\mathrm{a}}$ & $4.56 \pm 0.39^{\mathrm{a}}$ \\
\hline
\end{tabular}

${ }^{\mathrm{a}} \mathrm{P}<0.05$ vs. sham group, ${ }^{\mathrm{b}} \mathrm{P}<0.05$ vs. control group.

Sections were pre-incubated in a blocking solution containing 8\% normal donkey serum (SP-072-VX10, ImmunoReagents, Inc., Raleigh, NC, USA) in diluent solution (1\% bovine serum albumin (45496; EMD Millipore, Billerica, MA, USA) and $0.3 \%$ Triton $\mathrm{X}-100$ in $0.01 \mathrm{M}$ PBS, $\mathrm{pH} 7.5$ ) for $1 \mathrm{~h}$ at room temperature. Sections were subsequently incubated overnight at $4^{\circ} \mathrm{C}$ with the anti-HIF-1 $\alpha$ and anti-VEGF antibodies (both 1:500 in the diluent solution. Sections were washed three times in 0.01 M PBS for 10 min each and then incubated with the goat anti-mouse antibodies $(1: 1,000)$ diluted in $0.01 \mathrm{M}$ PBS for $1 \mathrm{~h}$ at room temperature in the dark. Sections were counterstained with toluidine blue (C0053; Shanghai Baoman Biotechnology Co., Ltd., Shanghai, China). Negative controls were treated with the secondary antibody alone. All samples were examined under a phase contrast microscope (08/357317, Olympus Corp., Tokyo, Japan.) and images were acquired using a digital camera and image-capturing software (both from Diagnostic Instruments, Inc., Sterling Heights, MI, USA).

Statistical analysis. The data were analyzed by one-way analysis of variance followed by Student-Newman-Keuls tests of multiple comparisons to determine whether there were significant differences between individual groups. $\mathrm{P}<0.05$ was considered to indicate a statistically significant difference.

\section{Results}

$B B B$ score measurement following operation. To verify whether SCI induction was successful, the BBB scores in the three groups were examined (Table I). There were no differences in the BBB scores among the three groups prior to operation. Immediately following the operation, the BBB scores in the three groups decreased to different extents. BBB scores in the SCI groups (both control and treatment groups) were significantly lower than in the sham group, suggesting that the SCI was successful $(\mathrm{P}<0.05)$. Following 1,3 and 7 days, BBB scores in the three groups had recovered to different extents and were significantly higher in the treatment group than in the control group $(\mathrm{P}<0.05)$. This suggests that administration of ML228 may alleviate SCI of the central nervous system and relieve associated symptoms.

$H \& E$ staining following operation. To further verify the success of SCI induction, H\&E staining of the spinal cord sections was performed. Under a high magnification (x200), the organizational structure of the spinal cord in the sham group was clear and there was no obvious cell damage in any of the groups (Fig. 1). However, there were obvious disorders and a loose arrangement of structure in the control and treatment groups. Moreover, clear blank areas were visible, suggesting the formation of glial scars (Fig. 1). Cell structures in the SCI groups were incomplete and the cells exhibited demyelinating changes, neuron vacuole degeneration and nuclear pyknosis. Furthermore, there were a large number of polymorphonuclear cells and extensive macrophage infiltration, and the control group exhibited edema and massive bleeding. However, compared with the control group, the structure in the treatment group was more ordered and clear, and fewer necrosis areas and glial scars were observed (Fig. 1). These results further verified the success of SCI induction and the effect of ML228 in relieving SCI.

Expression of HIF-1 $\alpha$ and VEGF in the spinal cord detected by western blotting. The expression of HIF-1 $\alpha$ and VEGF proteins in the spinal cord were measured by western blotting 1, 3 and 7 days following the operation. (Fig. 2A and B) Levels of HIF-1 $\alpha$ expression were significantly higher in the control and treatment groups compared with the sham group $(\mathrm{P}<0.05$; Fig. 2C). Furthermore, the expression of HIF-1 $\alpha$ in the treatment group was significantly higher than in the control group $(\mathrm{P}<0.05$; Fig. 2C). Similar results were observed regarding the expression of VEGF. In the control group, VEGF expression of control group was significantly higher than in the sham group $(\mathrm{P}<0.05)$ and expression of VEGF was significantly higher in the treatment group compared with the control group $(\mathrm{P}<0.05$; Fig. 2D).

Expression of HIF-1 $\alpha$ and VEGF in the spinal cord detected by immunohistochemistry. To investigate the HIF-1 $\alpha / \mathrm{VEGF}$ signaling pathway expression profiling of SCI in vivo, the distribution of HIF-1 $\alpha$ and VEGF expression was detected using immunohistochemistry. HIF-1 $\alpha$-positive cells were dyed lightest in color and were most highly dispersed in the sham group. Stronger dyeing of HIF-1 $\alpha$ was observed in the control group and the number of HIF-1 $\alpha$-positive cells was higher than in the sham group. The strongest and most prevalent HIF-1 $\alpha$ staining was observed in the treatment group (Fig. 3). This indicates that HIF-1 $\alpha$ expression increases following SCI, and that HIF-1 $\alpha$ expression levels are further increased by ML228 treatment. VEGF positive cells were dyed lightest in color and were highly dispersed in the sham group. Stronger dyeing of VEGF was observed in the control group and the number of VEGF positive cells was higher than in the sham group. The strongest and most prevalent VEGF staining was seen in the treatment group (Fig. 4). This indicates that VEGF expression increases following SCI and that levels of VEGF expression are further increased by ML228 treatment. 


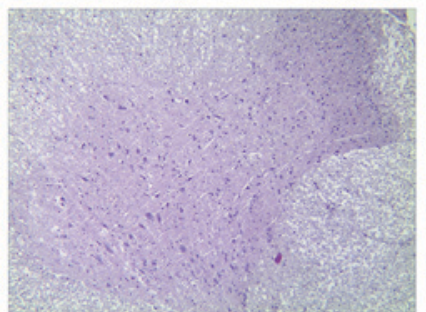

Sham group

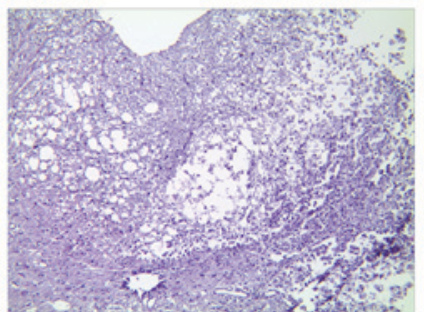

Control group

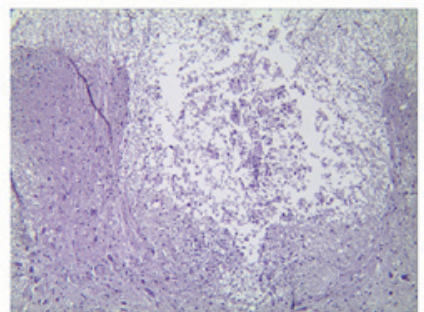

Treatment group

Figure 1. Hematoxylin and eosin staining of spinal cord tissue in the different groups 7 days after the operation was performed (magnification, x200).

A

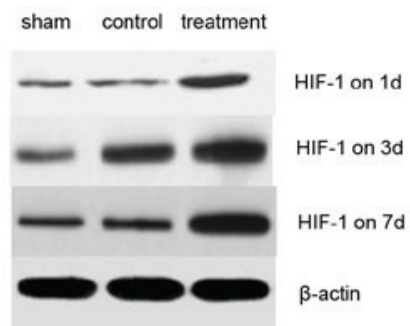

C

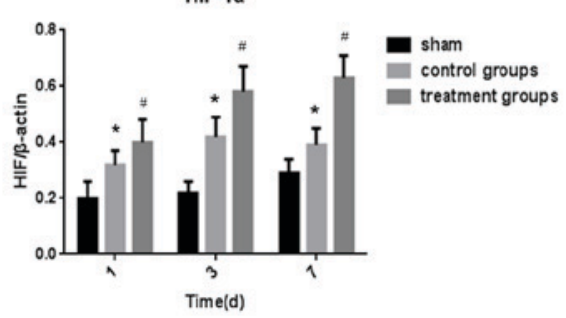

B

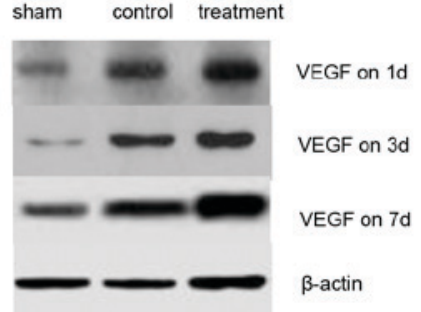

D

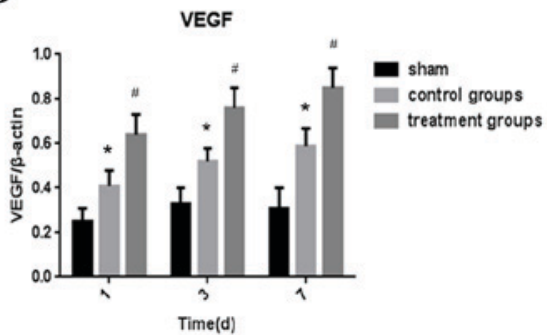

Figure 2. Levels of expression of the HIF-1 and VEGF proteins. (A and B) Western blot analysis of HIF-1 and VEGF expression in the spinal cord. Densitometric analysis of (C) HIF-1 and (D) VEGF abundance in protein lysates of the spinal cord. VEGF, vascular endothelial growth factor; HIF-1, hypoxia inducible factor 1 ; d, day(s). ${ }^{*} \mathrm{P}<0.05$ vs. sham group; ${ }^{*} \mathrm{P}<0.05$ vs. sham and control group.

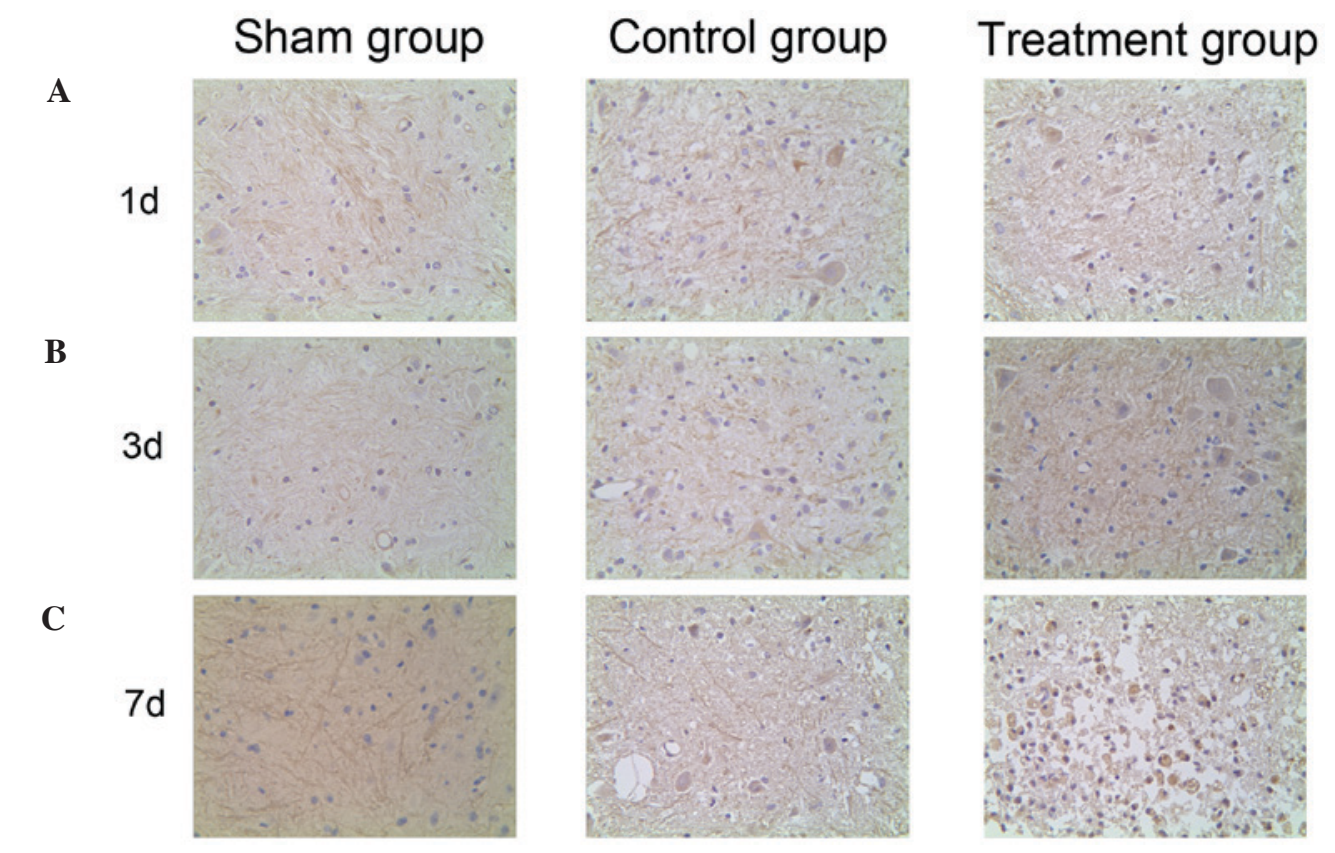

Figure 3. Hypoxia inducible factor-1 $\alpha$ expression pattern in spinal cord in different groups (A) $1 \mathrm{~d}$, (B) $3 \mathrm{~d}$ and (C) $7 \mathrm{~d}$ after operation (magnification, $\mathrm{x} 400$ ). $\mathrm{d}$, day(s). 


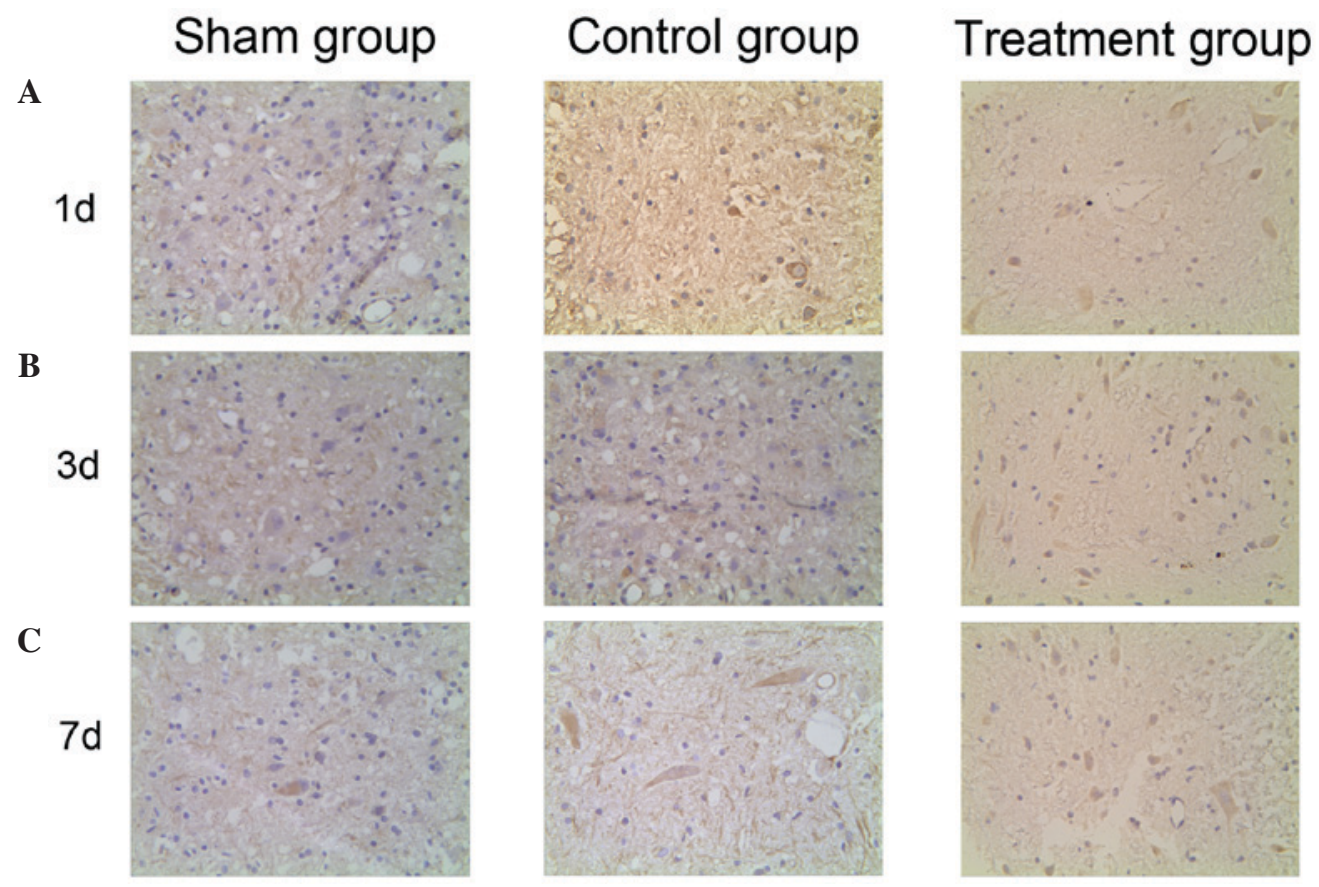

Figure 4. Vascular endothelial growth factor expression pattern in spinal cord in the different groups (A) $1 \mathrm{~d}$, (B) $3 \mathrm{~d}$ and (C) $7 \mathrm{~d}$ after operation (magnification, x400). d, day(s).

\section{Discussion}

At present, SCI is a medical problem worldwide; figures indicate that the morbidity of SCI is 20-40 per million (13). Due to improvements in treatment, the mortality rate from SCI has decreased from $\sim 50 \%$ in the early 20 th century to $6 \%$ at present; however, the recovery from nerve damage remains poor.

HIF-1 is a transcriptional activator consisting of the oxygen regulation subunit HIF-l $\alpha$ and the structural subunit HIF-1 $\beta$, and regulates the expression of genes following changes in oxygen concentrations within cells (14). HIF-l $\alpha$ serves a crucial role in the regulation of multiple equilibrium in hypoxia. It is a transcription factor that improves the adaptation of cells and tissues to ischemic and hypoxic environments (15) and activates the transcription of a number of factors, including erythropoietin and VEGF (16). Recently, it has been demonstrated that HIF-1 is widely involved in the adaption to hypoxia in mammalian cells. It is an important transcriptional regulator of the cellular adaptation to hypoxia and is associated with a number of diseases (17). VEGF was initially identified as an endothelial specific growth factor, however it has been since been demonstrated that VEGF has a variety of different functions $(18,19)$. VEGF is the most powerful angiogenesis promoting factor, which can directly act on vascular endothelial cells, stimulate the proliferation and migration of vascular endothelial cells, and promote angiogenesis (20). Studies have suggested that VEGF is a type of nerve protection factor that acts by a separate mechanism to protect nerve cells and promote the survival of endothelial cells in an anti-apoptotic way, not only in angiogenesis $(21,22)$. Furthermore, studies have demonstrated that, as well as having a nervous protective effect, VEGF has the ability to promote nerve regeneration and stimulate the growth and survival of neurons, glial cells and axons (23-25). HIF-1 is the upstream regulatory protein of VEGF transcription, which is itself upregulated by ischemia or hypoxia. The role of VEGF in various tumors and hypoxic ischemic heart disease is clear, however only a limited number of studies into the role of VEGF in spinal cord injury have been performed. Therefore, the present study investigated ML228, a known HIF-1/VEGF activator, and tested whether the activation of the HIF-1/VEGF signaling pathway was able to improve recovery from SCI.

The results demonstrated that the functional recovery, measured using the BBB scoring system, was improved by treatment with ML228. In addition, very low expression of HIF-1 $\alpha$ and VEGF was detected in the spinal cord of the sham group. However, expression of these proteins significantly increased following SCI, indicating that a large number of HIF-1 and VEGF proteins are produced in order to adapt to the hypoxic ischemia induced by SCI in rats. Subsequently, as expected, following administration of the HIF-1/VEGF signaling pathway activator to the treatment group, HIF-1 $\alpha$ and VEGF expression increased compared with the control group, suggesting that HIF-1/VEGF activation contributed to recovery from SCI.

The reduction of blood flow in the spinal cord is the primary cause of necrosis and nervous dysfunction (26). Regardless of the degree of trauma, ischemia and infarction appear in the gray matter 1-2 $\mathrm{h}$ after SCI, while the extent of trauma in the white matter is related to changes in blood flow (27). The severity of nerve dysfunction is determined by the ischemia extent of the white matter, and ischemia is one of the primary causes of secondary spinal cord injury (28). Mahon et al (29) demonstrated that ischemia and hypoxia of the spinal cord occur immediately following SCI, and determined that microcirculation changes following injury are a key mechanism of secondary damage. Therefore, other scientists have concluded 
that hypoxia may be a useful technique for tissue specific gene therapy, as it has reduced side effects and increased efficacy (30). Long et al (31) determined that in hypoxic conditions following SCI, the upregulation of VEGF is consistent with increasing HIF-1 $\alpha$ in acute periods, in accordance with the results of the current study. Furthermore, there is a correlation between VEGF expression and angiogenesis (protecting vascular endothelial cells, increasing blood vessel density and improving regional blood flow), neurogenesis (antiapoptosis, neurotrophy and attenuating axonal degradation), and locomotor ability improvement $(32,33)$.

In conclusion, intervening to increase the activity of the HIF-1/VEGF signaling pathway by administrating ML228 following SCI may improve the local hypoxic ischemia environment, reduce SCI secondary injury and promote the recovery of neurological function. Further studies on the mechanism of SCI are required and may provide novel therapeutic strategies for future SCI treatment.

\section{References}

1. Anwar MA, Al Shehabi TS and Eid AH: Inflammogenesis of secondary spinal cord Injury. Front Cell Neurosci 10: 98, 2016.

2. Schwab JM, Zhang Y, Kopp MA, Brommer B and Popovich PG: The paradox of chronic neuroinflammation, systemic immune suppression, autoimmunity after traumatic chronicspinal cord injury. Exp Neurol 258: 121-129, 2014.

3. Jeong W, Bazer FW, Song G and Kim J: Expression of hypoxia-inducible factor-1 by trophectoderm cells in response to hypoxia and epidermal growth factor. Biochem Biophys Res Commun 469: 176-182, 2016.

4. Stampas A and Tansey KE: Spinal cord injury medicine and rehabilitation. Semin Neurol 34: 524-533, 2014.

5. Gui L, Liu B and Lv G: Hypoxia induces autophagy in cardiomyocytes via a hypoxia-inducible factor 1-dependent mechanism. Exp Ther Med 11: 2233-2239, 2016.

6. Kimura H, Weisz A, Ogura T, Hitomi Y, Kurashima Y, Hashimoto K, D'Acquisto F, Makuuchi M and Esumi $\mathrm{H}$ : Identification of hypoxia-inducible factorl ancillary sequence and its function in vascular endothelial growth factor gene induction by hypoxia and nitric oxide. J Biol Chem 276: 2292-2298, 2001.

7. Savas S, Savas C, Altuntas I and Adiloglu A: The correlation between nitric oxide and vascular endothelial growth factor in spinal cord injury. Spinal Cord 46: 113-117, 2008.

8. Xing J and Lu J: HIF-1 $\alpha$ activation attenuates IL-6 and TNF- $\alpha$ pathways in hippocampus of rats following transient global ischemia. Cell Physiol Biochem 39: 511-520, 2016.

9. Theriault JR, Felts AS, Bates BS, Perez JR, Palmer M, Gilbert SR, Dawson ES, Engers JL, Lindsley CW and Emmitte KA: Discovery of a new molecular probe ML228: An activator of the hypoxia inducible factor (HIF) pathway. Bioorg Med Chem Lett 22: 76-81, 2012.

10. Basso DM, Beattie MS and Bresnahan JC: A sensitive and reliable locomotor rating scale for open filed testing in rats. Neurotrauma 12: 1-21, 1995.

11. Scheff SW, Saucier DA and Cain ME: A statistical method for analyzing rating scale data: The BBB locomotor score. J Neurotrauma 19: 1251-1260, 2002.

12. Lu P, Graham L, Wang Y, Wu D and Tuszynski M: Promotion of survival and differentiation of neural stem cells with fibrin and growth factor cocktails after severe spinal cord injury. J Vis Exp: e50641, 2014.

13. Chavez JC and LaManna JC: Activation of hypoxia-inducibled factor-1 in the rat cerebral cortex after transient global ischemia: Potential role of insulin-like growth faetor-1. J Neurosci 22: 8922-8931, 2002.

14. Linke S, Stojkoski C, Kewley RJ, Booker GW, Whitelaw ML and Peet DJ: Substrate reqnirements of the oxygen-sensing asparaginyl hydroxylase factor inhibiting hypoxia-inducible factor. J Bid Chem 279: 14391-14397, 2004.
15. Jantsch J and Schödel J: Hypoxia and hypoxia-inducible factors in myeloid cell-driven host defense and tissue homeostasis. Immunobiology 220: 305-314, 2015.

16. Manalo DJ, Rowan A, Lavoie T, Natarajan L, Kelly BD, Ye SQ, Garcia JG and Semenza GL: Transcriptional regulation of vascular endothelial cell responses to hypoxia by HIF-1. Blood 105: 659-669, 2005.

17. Zimna A and Kurpisz M: Hypoxia-Inducible Factor-1 in physiological and pathophysiological Angiogenesis: Applications and therapies. Biomed Res Int 2015: 549412, 2015.

18. Basagiannis D, Zografou S, Murphy C, Fotsis T, Morbidelli L, Ziche M, Bleck C, Mercer J and Christoforidis S: VEGF induces signalling and angiogenesis by directing VEGFR2 internalisation via macropinocytosis. J Cell Sci pii: jes: 188219, 2016 (Epub ahead of print)

19. Vasta S, Di Martino A, Zampogna B, Torre G, Papalia R and Denaro V: Role of VEGF, nitric oxide, and sympathetic neurotransmitters in the pathogenesis of tendinopathy: A review of the current evidences. Front Aging Neurosci 8: 186, 2016.

20. D'Alessio A, Proietti G, Lama G, Biamonte F, Lauriola L, Moscato U, Vescovi A, Mangiola A, Angelucci C and Sica G: Analysis of angiogenesis related factors in glioblastoma, peritumoral tissue and their derived cancer stem cells. Oncotarget 2016 (Epub ahead of print).

21. Ortuzar N, Argandoña EG, Bengoetxea $H$ and Lafuente JV: Combination of intracortically administered VEGF and environmental enrichment enhances brain protection in developing rats. J Neural Transm (Vienna) 118: 135-144, 2011.

22. Tovar-Y-Romo LB and Tapia R: VEGF protects spinal motor neurons against chronic excitotoxic degeneration in vivo by activation of PI3-K pathway and inhibition of p38MAPK. J Neurochem 115: 1090-1101, 2010.

23. Song S, Park JT, Na JY, Park MS, Lee JK, Lee MC and Kim HS: Early expressions of hypoxia-inducible factor 1alpha and vascular endothelial growth factor increase the neuronal plasticity of activated endogenous neural stem cells after focal cerebral ischemia. Neural Regen Res 9: 912-918, 2014.

24. Ding XM, Mao BY, Jiang S, Li SF and Deng YL: Neuroprotective effect of exogenous vascular endothelial growth factor on rat spinal cord neurons on vitro hypoxia. Chin Med J(Engl) 118: 1644-1650, 2005.

25. Jin K, Mao XO and Greenberg DA: Vascular endothelial growth factor stimulates neurite outgrowth from cerebral cortical neurons via Rho kinase signaling. J Neurobiol 66: 236-242, 2006.

26. Lu K, Liang CL, Chen HJ, Chen SD, Hsu HC, Liliang PC, Lin TK and Cho CL: Injury severity and cell death mechanisms: Effects of concomitant hypovolemic hypotension on spinal cord ischemia-reperfusion in rats. Exp Neurol 185: 120-132, 2004.

27. Natarajan R, Salloum FN, Fisher BJ, Kukreja RC and Fowler AA III: Hypoxia inducible factor-1 upregulates adiponectin in diabetic mouse hearts and attenuates post-ischemic injury. J Cardiovasc Pharmacol 51: 178-187, 2008.

28. Tamosaityte S, Galli R, Uckermann O, Sitoci-Ficici KH, Later R, Beiermeister R, Doberenz F, Gelinsky M, Leipnitz E, Schackert G, et al: Biochemical monitoring of spinal cord injury by FT-IR spectroscopy-effects of therapeutic alginate implant in rat models. PLoS One 10: e0142660, 2015.

29. Mahon PC, Hirota K and Semenza GL: FIH·1: A novel protein that interacts with HIF-lalpha transcriptional activivity. Genes Dev 15: 2675-2686, 2005.

30. Rhim T, Lee DY and Lee M: Hypoxia as a target for tissue specific gene therapy. J Control Release 172: 484-494, 2013.

31. Long HQ, Li GS, Hu Y, Wen CY and Xie WH: HIF-1 $\alpha$ /VEGF signaling pathway may play a dual role in secondary pathogenesis of cervical myelopathy. Med Hypotheses 79: 82-84, 2012.

32. Herrera JJ, Nesic O and Narayana PA: Reduced vascular endothelial growth factor expression in contusive spinal cord injury. J Neurotrauma 26: 995-1003, 2009.

33. Liu Y, Figley S, Spratt SK, Lee G, Ando D, Surosky R and Fehlings MG: An engineered transcription factor which activates VEGF-A enhances recovery after spinal cord injury. Neurobiol Dis 37: $384-393,2010$ 